\title{
The dynamical state of a thick cloudy torus around an AGN
}

\author{
T. Beckert ${ }^{1}$ and W. J. Duschl ${ }^{2,1}$ \\ 1 Max-Planck-Institut für Radioastronomie, Auf dem Hügel 69, 53121 Bonn, Germany \\ e-mail: tbeckert@mpifr-bonn.mpg.de \\ ${ }^{2}$ Institut für Theoretische Astrophysik der Universität Heidelberg, Tiergartenstraße 15, 69121 Heidelberg, Germany
}

Received 25 February 2004 / Accepted 6 July 2004

\begin{abstract}
We present stationary models of obscuring dusty tori around active galactic nuclei. Vertical and radial structure are discussed separately based on a dynamical approach to a cloudy accretion flow. The vertical cloud distribution is described by a modified isothermal disk for arbitrary thickness of the torus with a wide funnel along the symmetry axis. The radial accretion flow is a consequence of cloud-cloud collisions. Accretion in the combined gravitational potential of central black hole and stellar cluster generates free energy, which is dissipated in collisions, and maintains the thickness of the torus. We focus on the largest clouds at the tidal shear limit, which will dominate the dynamics and the appearance of torus. Successful obscuration as required by unified schemes of AGN is achieved for large mass accretion rates above the Eddington limit of the central black hole. A complete quantitative treatment for the putative torus in the prototypical Seyfert 2 nucleus of NGC 1068 is given.
\end{abstract}

Key words. accretion, accretion disks - black hole physics - ISM: clouds - galaxies: active - galaxies: nuclei galaxies: individual: NGC 1068

\section{Introduction}

Based on the pioneering work of Miller and Antonucci (Miller \& Antonucci 1983; Antonucci \& Miller 1985), today the major difference between Seyfert 1 and Seyfert 2 galaxies is attributed to orientation effects, caused by an optically thick torus around a super-massive black hole. According to the original unification scheme all Seyfert 2 nuclei harbor a Seyfert 1 core. A further distinction between true type 2 cores, where there is no need for obscuration, and the obscured type $1 \mathrm{~s}$, which appear as type $2 \mathrm{~s}$ is required. These true type 2 Seyferts seem to be exclusively low-luminosity systems and most samples are likely to be biased against those objects. The observed ratio of Seyfert 2 to Seyfert 1 galaxies of about 4 (Maiolino \& Rieke 1995) would imply a relative torus thickness of $H / R=x_{H} \approx 1.33(R$ is the distance from the black hole and $H$ the thickness of the torus at that location), if the distinction is solely due to the orientation of the torus. Because Seyfert 1s are predominately seen in face-on galaxies, the obscuration may partially be due to dust in the galactic disk. If the orientation of the torus is unrelated to the orientation of the galaxy, the torus thickness $x_{H} \approx 1.33$ is to be seen as an upper limit. A discussion of biases in different samples of Seyfert galaxies can be found in Alonso-Herrero et al. (2003). It appears that effects of the host galaxy and selection effects against low-luminosity systems are difficult to account for quantitatively. While the overall picture seems to be successful (see, e.g., Schmitt et al. 2001), both, statistics over larger ensembles of active galaxies, and spectral properties of individual objects indicate that a simple, smooth torus is an oversimplification of the actual situation (Tran 2001, and references therein). This unification scheme is thought to apply to AGN in general, and not only to Seyfert galaxies. Type 1 objects are those in which a direct view towards the immediate vicinity of the active nucleus is possible, while in type 2 objects, the direct view is impeded by the obscuring torus and the light from the nucleus proper can be seen only through reflection.

Since more than a decade, clumpy models for the circumnuclear torus are under discussion (Pier \& Krolik 1992, 1993; Rowan-Robinson 1995; Granato et al. 1997; Nenkova et al. 2002). It has been realized that a fit of the IR SED alone is not sufficient to determine the various parameters of the different models suggested. For the scenario of well separated clouds Nenkova et al. (2002) derived the required properties of the torus and the clouds embedded in it as far as they are constrained by the SED.

In this paper we present a model for such a nonselfgravitating cloudy torus around a black hole and cospatial with a nuclear stellar cluster. We derive hydrodynamically consistent physical properties of the individual clouds, as well as the global structure of the torus. Finally, we compare the results to observations of the prototypical Seyfert 2 galaxy NGC 1068 and with the requirements derived by Nenkova et al. (2002).

\section{The scenario of a cloudy torus}

The basic ideas of our model for a clumpy torus around an AGN are described in Vollmer et al. (2004). The torus is 
thought to consist of a large number of distinct, cold and dusty clouds. The dynamics of the cloud distribution is governed by cloud-cloud collisions and accompanied by accretion towards the AGN. In these direct cloud-cloud collisions part of the kinetic energy of the clouds is dissipated, thermalized, and radiated away. These collisions also allow a redistribution of the angular momentum of clouds and can lead to both an outward transport of angular momentum and inward mass accretion in the combined gravitational potential of black hole and central star cluster. In an equilibrium state, where no mass is lost from or acquired vertically by the torus, the radial mass accretion rate is

$\dot{M}=2 \pi \Sigma v_{\text {acc }}$,

where $v_{\text {acc }}$ is the vertically averaged radial accretion velocity of clouds. Here we neglect mass locked up in stars by star formation and the regain from stellar winds. In Vollmer et al. (2004) we argued that clouds in a torus are quasi-stable and created or destroyed in cloud collisions. Star formation and stellar feedback appears to be unimportant, because the accretion timescale is usually shorter than the star formation time-scale and the momentum transfer from supernovae and stellar winds is inefficient for quasi-stable clouds.

In an equilibrium state, local quantities like mass density $\rho$, sound speed in clouds $c_{\mathrm{s}}$, or velocity dispersion $\sigma$ are meaningful quantities when averaged for a dynamical time-scale $\Omega^{-1}=$ $H / \sigma$. Substantial changes are expected on a feeding time scale, when the mass supplied at an outer boundary from the galactic disk varies. The outer boundary is determined either by the transition to a multi-phase ISM, where molecular clouds appear from and dissolve into other phases of the ISM, or where other mechanisms than cloud collisions like bar modes create a radial mass inflow.

For the effective viscosity from cloud-cloud collisions we modify our model of quasi-stable clouds developed in Vollmer et al. (2004) for a local description of the torus. We follow Goldreich \& Tremaine (1978) and use

$v=\frac{\tau}{1+\tau^{2}} \frac{\sigma^{2}}{\Omega}$

for the viscosity. Here $\tau=\Omega / \omega_{\text {coll }}$ is the dimensionless collision time. The viscosity reaches its maximum at $\tau=1$ and its functional form is symmetric with respect to $\tau \rightarrow 1 / \tau$. So Eq. (2) looks the same when formulated in terms of the dimensionless collision frequency $\varpi=\tau^{-1}=\omega_{\text {coll }} / \Omega$. The absolute value of the viscosity for a densely populated torus $(\tau \sim 1)$ is quantitatively relevant in our context. Vertical hydrostatic equilibrium $H=\sigma / \Omega$ and the collision frequency $\omega_{\text {coll }}=\sigma / l_{\text {coll }}$ for a velocity dispersion $\sigma$ and mean free path $l_{\text {coll }}$ can be used to see that $l_{\text {coll }}=H \tau$. The frequency $\varpi$ plays the role of a vertical optical depth for intercepting a cloud.

The torus resides in a well specified gravitational potential, where we can make use of the Toomre parameter defined as

$Q=\frac{\sigma K}{\pi G \Sigma}$.

Here the epicycle frequency $\kappa$ appears, which is relevant in the transition region from the sphere of influence of the central point mass to the region dominated by the star cluster. The stability criterion of Toomre applies to thin disks and the geometrically thick torus is well in the stable regime, so we use $Q$ to relate velocity dispersion and surface density. The assumption that clouds in the torus are mostly at the shear limit (Vollmer et al. 2004) $\rho_{\mathrm{Cl}} / \rho_{\text {crit }}=4 /\left(3 Q \phi_{V}\right)$, with $\phi_{V}$ the volume filling factor of clouds, gives then an upper limit for $Q$. This property makes $Q$ valuable in our discussion.

\subsection{Cloud properties}

Cold, molecular and dusty clouds are the basic building blocks of the torus model. In spite of the complexity of their internal physics, we regard them as self-gravitating, spherical clouds, which are sufficiently described by their radius $r_{\mathrm{Cl}}$ and internal sound speed $c_{\mathrm{s}}$. Photodisoziation fronts, non-isothermal structure, disturbed shapes due to collisions or tidal forces are neglected in this approach. The mean free path of these clouds is directly linked to their volume filling factor $\phi_{V}$, which is determined by the average size of clouds. We can assume that clouds are slightly self-gravitating, which implies that the clouds freefall time and the sound crossing time are approximately equal. This leads to an expression

$\phi_{V}=\frac{32 G \rho_{0} r_{\mathrm{Cl}}^{2}}{3 \pi c_{\mathrm{s}}^{2}}$

in the torus midplane. The mean density in the midplane $\rho_{0}$ and the surface density used in the definition of $Q$ in Eq. (3) are related by the vertical stratification of the model. In a more general approach we assume a linear dependence of surface density $\Sigma$ on $\rho_{0}$ and $H$, so that we write $\Sigma=\xi \rho_{0} H$. For the particular model of Sect. 3.2 we discuss this relation in Sect. 3.4 in more detail. With the value $\xi=\sqrt{2 \pi}$ from Eq. (23) we find the mean free path

$l_{\mathrm{coll}}=\frac{\pi^{2} \xi Q}{8 \tilde{\rho}} \frac{c_{\mathrm{s}}^{2}}{\kappa \Omega r_{\mathrm{Cl}}}$

where $\tilde{\rho}=\rho(z) / \rho_{0}$ is the normalized vertical cloud distribution. Using the optical depth in the midplane we get $l_{\text {coll }}=\tau \sigma /(\tilde{\rho} \Omega)$ and the average cloud radius, which we assume to be constant throughout the vertical structure, is

$r_{\mathrm{Cl}}=\frac{\pi^{2} \xi}{8} \frac{Q}{\tau} \frac{c_{\mathrm{s}}^{2}}{\kappa \sigma}$

Without referring to the accretion process and the viscosity we have parameterized the mean free path of clouds and their radius. It is the velocity dispersion, resulting from the angular momentum transport, which connects the cloud properties to the accretion process. Independently we can set a limit on $Q$ and determine the surface density parameter $\xi$ more precisely.

\subsection{Shear limit}

The appearance of the torus in radiative transfer calculations will be dominated by the largest clouds, because they contribute most to the reduction of the mean free path $l_{\text {coll }}=$ $\left(\pi n r_{\mathrm{Cl}}^{2}\right)^{-1}$ ( $n$ is the cloud number density). But these large 
clouds are more likely to be disrupted by tidal forces than smaller clouds and we follow the analysis of Vollmer et al. (2004) with the modifications described above. For a given sound speed $c_{\mathrm{s}}$ Eq. (6) can be used to express the tidal disruption limit as a limit for $Q$ instead of the cloud size or the cloud density in the form

$Q \leq \frac{\sqrt{8} \tau}{\pi \xi} \frac{\sigma}{c_{\mathrm{s}}} \sqrt{\frac{\kappa}{\Omega}}$

The shear limit in this form is independent of angular momentum or energy transport through the torus.

\section{The Vertical stratification of geometrically thick disks}

The appearance of a thick disk or torus in radiative transfer calculations does depend on their vertical structure. It must therefore be treated with more care than in the case of thin disks. The vertical structure of geometrically thin disks in the potential of a point mass can be simplified by using the linear term in an expansion of the potential in the vertical direction around the midplane of the disk. Vertical velocity gradients $v_{z}\left(\partial v_{z} / \partial z\right)$ are always much smaller than the pressure gradient supporting the vertical structure of thin disks and the hydrostatic balance in the vertical direction in cylindrical coordinates is given by

$\frac{1}{\rho} \frac{\partial p}{\partial z}=-\frac{G M}{R^{3}} z$

where $R$ is the radius from the central mass $M$ in the disk plane (radius in cylindrical coordinates).

Whenever a disk or torus becomes geometrically thick so that the disk scale height $H=\sqrt{p_{0} / \rho_{0}} / \Omega_{K}$ is not small compared to the radius $R$ anymore, the linear term in the expansion of the potential gives a much too steep gradient of that potential to be balanced by the pressure in the torus. It is less obvious for a thick torus that vertical velocity gradients can be neglected. Nonetheless we assume that the bulk velocity $v_{z}$ is much smaller than the velocity dispersion $\sigma$ in the torus. The velocity dispersion of an ensemble of cool clouds, which comprise the torus gives rise to a dynamical pressure $p$.

One may describe the ensemble of clouds by a polytropic equation of state $p=K \rho^{\gamma}$. This includes the case of the dispersion $\sigma$ being independent $(\gamma=1)$ of the cloud density $\rho=\phi_{V} \rho_{\mathrm{cl}}$, where $\rho_{\mathrm{cl}}$ is the mean density of an individual cloud and $\phi_{V}$ the volume filling factor of these clouds. The vertical hydrostatic balance becomes an equation for the density

$\frac{\partial \rho}{\partial x}=-a \rho^{2-\gamma} \frac{x}{\left(1+x^{2}\right)^{3 / 2}}$

with the parameter

$a=(K \gamma)^{-1} \frac{G M}{R}$

and the rescaled vertical coordinate $x=z / R$. The density distribution for vertical, hydrostatic equilibrium (Eq. (9)) in the potential of a central point mass has analytic solutions for $\gamma \geq 1$. For $\gamma>1$ all polytropic solutions have a finite height $x_{H}$, where the density reaches zero. We will only give the result for the isothermal case and discuss a modified version for general potentials in Sect. 3.2.

\subsection{The isothermal torus}

For a velocity dispersion independent of vertical position and density the torus is equivalent to an isothermal disk. For the corresponding polytropic index $\gamma=1$ the hydrostatic balance is easily solved by rewriting Eq. (9)

$\frac{\partial \ln \rho}{\partial x}=-a \frac{x}{\left(1+x^{2}\right)^{3 / 2}}$

The integration of Eq. (11) gives

$\ln \left(\frac{\rho}{\rho_{0}}\right)=-\frac{a\left(\sqrt{1+x^{2}}-1\right)}{\sqrt{1+x^{2}}}$,

where $\rho_{0}$ is the density in the torus midplane and the density decreases exponentially. The parameter $a$ is the squared ratio of the Keplerian circular orbital velocity and the velocity dispersion with $K=\sigma^{2}$. This special case may be appropriate for a torus where cloud collisions are not too frequent. In reality AGN produce outflows along the polar axes, which are probably collimated by the torus. The interaction of the torus with the outflow provides a finite pressure and a vertical cut-off height, where gas at larger heights is dynamically not part of the torus anymore but belongs to the outflow.

\subsection{The modified isothermal torus}

The inclusion of a cut-off height and the structure in a general potential can be modeled with a collisionless (lowered ${ }^{1}$ ) isothermal distribution of clouds in an external potential. In analogy to the development of King models exemplified by Binney \& Tremaine (1987) we assume a distribution function of clouds in phase space of the form

$f=\left\{\begin{array}{cc}\frac{\rho_{1}}{\left(2 \pi \sigma^{2}\right)^{3 / 2}}\left(\mathrm{e}^{\mathcal{E} / \sigma^{2}}-1\right) & \text { for } \mathcal{E}>0 \\ 0 & \text { for } \mathcal{E} \leq 0\end{array}\right.$

This distribution is constructed like a Maxwell-Boltzmann distribution with a specific energy $\mathcal{E}=\Psi-v^{2} / 2$ where $v$ is the velocity of individual clouds and

$\Psi=-\Phi(r)+\Phi\left(r_{H}\right)=\frac{G \tilde{M}(r)}{R \sqrt{1+x^{2}}}-\frac{G \tilde{M}\left(r_{H}\right)}{R \sqrt{1+x_{H}^{2}}}$

a potential constructed in such a way ${ }^{2}$ that $f$ vanishes at vertical distances larger than a presumed disk height $H=x_{H} R$. We use $r$ as the radius in spherical coordinates and $r_{H}$ the cut-off radius corresponding to the cylindrical radius $R$. The velocities allowed for clouds lie in a range $|v| \in[0, \sqrt{2 \Psi}]$ and the upper

\footnotetext{
${ }^{1}$ The mean velocity of clouds in the central plane of the torus is lower than the virial velocity there.

2 Note that integrating the mass density for an $\alpha$-model (see Eq. (18)) of a quasi-isothermal stellar cluster gives an enclosed mass of $M(r) \sim 4 \pi \rho_{\star, \mathrm{c}} R_{\mathrm{c}}^{3}\left(r / R_{\mathrm{c}}\right)^{(3-\alpha)}$ within a radius $r=R \sqrt{1+x^{2}}$ larger than the core radius $R_{\mathrm{c}}$. The contribution to the potential $\Psi$ is $\Psi_{\text {cluster }}=$ $(\alpha-2) G M(r) / r$. The factor $(\alpha-2)$, which is often negative, indicates that it is not simply the total enclosed mass which must be inserted in Eq. (14) for the potential. This is in contrast with calculating the gravitational force where the enclosed mass is sufficient.
} 
limit changes with the potential $\Psi$ and gets successively narrower when $z \rightarrow H$. The local density due to clouds is

$$
\begin{aligned}
\rho & =4 \pi \int_{0}^{\sqrt{2 \Psi}} \mathrm{d} v v^{2} f \\
& =\rho_{1}\left(\mathrm{e}^{\Psi / \sigma^{2}} \operatorname{erf}(\sqrt{\Psi} / \sigma)-\frac{2}{\sqrt{\pi}} \frac{\sqrt{\Psi}}{\sigma}\left(1+\frac{2 \Psi}{3 \sigma^{2}}\right)\right)
\end{aligned}
$$

where $\operatorname{erf}(x)$ is the error-function (Abramowitz \& Stegun 1972). For self-gravitating spherical systems one derives a differential equation for these King models, but in our case the potential is already specified by Eq. (14), where the mass $\tilde{M}(r)$ is provided by the central black hole and a nuclear, stellar cluster. The density given by Eq. (15) can be interpreted either as mass density distribution or the number density of clouds. For a given external potential the solution in Eq. (15) is a two parameter $\left(\sigma, x_{H}\right)$ family of density distributions $\rho_{\sigma, x_{H}}(z)$. The square of the true one dimensional velocity dispersion of this model is

$\overline{v^{2}}=3 \sigma^{2} \frac{\mathrm{e}^{\Psi / \sigma^{2}} \operatorname{erf}(\sqrt{\Psi} / \sigma)-\frac{2}{\sqrt{\pi}} \frac{\sqrt{\Psi}}{\sigma}\left(1+\frac{2 \Psi}{3 \sigma^{2}}+\frac{4 \Psi^{2}}{15 \sigma^{4}}\right)}{\mathrm{e}^{\Psi / \sigma^{2}} \operatorname{erf}(\sqrt{\Psi} / \sigma)-\frac{2}{\sqrt{\pi}} \frac{\sqrt{\Psi}}{\sigma}\left(1+\frac{2 \Psi}{3 \sigma^{2}}\right)}$

and is not to be confused with the velocity $\sigma$, which we nonetheless call velocity dispersion in this paper. For a parameter pair $\left(\sigma, x_{H}\right)$, the density $\rho_{0}$ at the disk midplane

$\rho_{0}=\rho_{1}\left(\mathrm{e}^{y^{2}} \operatorname{erf}(y)-\frac{2}{\sqrt{\pi}} y\left(1+\frac{2}{3} y^{2}\right)\right)$,

depends on the parameter $y=\sqrt{\Psi(x=0)} / \sigma$ and can be used to normalize the constant $\rho_{1}$ in the density distribution of Eq. (15). In the case of a large torus thickness $x_{H}=1.33$ and a moderate velocity dispersion corresponding to $y=5$ in the potential of a $1.2 \times 10^{7} M_{\odot}$ black hole and an almost isothermal star cluster with $\alpha=2.1$ and a core radius of $R_{\mathrm{c}}=0.32 \mathrm{pc}$, which becomes dynamically important for $R>2$ pc, we plot iso-density contours in Fig. 1. The radial density profile is derived in Sect. 4.1 (Eq. (30)) with $\rho_{0} \propto R^{-2.5}$.

\subsection{The potential for an $\alpha$-model of a stellar cluster with a central black hole}

For a spherically symmetric star cluster we can use an $\alpha$-model for the density distribution which determines the gravitational potential $\Phi$. The model is described by a central density $\rho_{*, \mathrm{c}}$, a core radius $R_{\mathrm{c}}$ and a power-law slope $\alpha$ in the form

$\rho(r)=\frac{\rho_{*, \mathrm{c}}}{\sqrt{1+\left(r / R_{\mathrm{c}}\right)^{2 \alpha}}}$

The enclosed mass within a radius $r$ is

$M(r)=\frac{4}{3} \pi \rho_{*, \mathrm{c}} r_{2}^{3} F_{1}\left(\frac{3}{2 \alpha}, \frac{1}{2}, 1+\frac{3}{2 \alpha},-\left(\frac{r}{R_{\mathrm{c}}}\right)^{2 \alpha}\right)$.

where ${ }_{2} F_{1}$ is a hypergeometric function, which has a series expansion leading to a reasonable approximation for the enclosed mass outside the core radius of

$M(r)=\frac{4}{3} \pi \rho_{*, \mathrm{c}} R_{\mathrm{c}}^{3}\left[\frac{\Gamma\left(1+\frac{3}{2 \alpha}\right) \Gamma\left(\frac{1}{2}-\frac{3}{2 \alpha}\right)}{\sqrt{\pi}}+\frac{\left(r / R_{\mathrm{c}}\right)^{3-\alpha}}{1-\alpha / 3}\right]$.

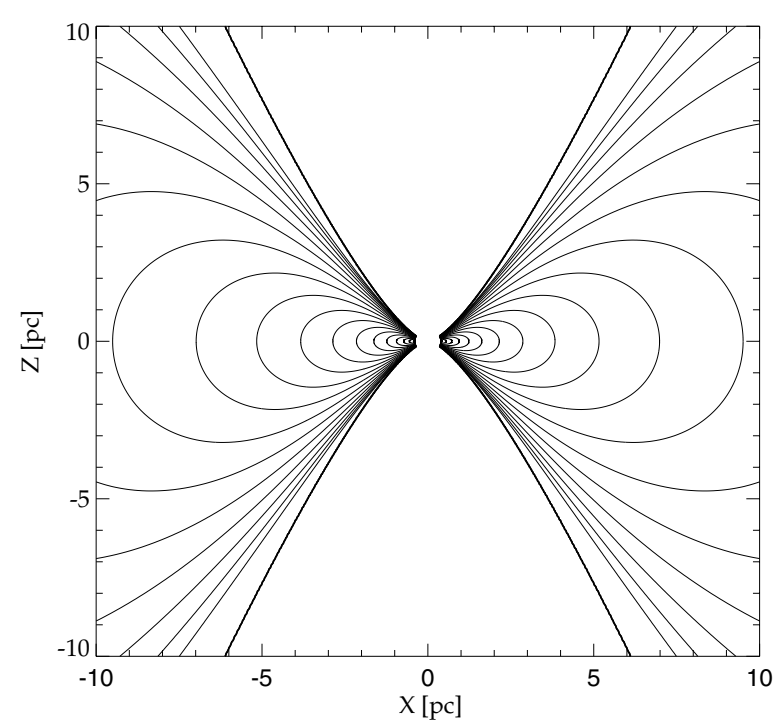

Fig. 1. Mass density contours in the meridional plane of the torus model. Contour levels are stepping down a factor of 2 from the peak in density. The model assumes $x_{H}=\sqrt{2 \pi} H /(0.56 R)$ implied by the discussion at the end of Sect. 3.4. The vertical collision depth is set to $\tau=1$ and the largest clouds are at the shear limit (Eq. (27)). The potential described in Sect. 6 for NGC 1068 is used and the inner radius for dust sublimation is set to $1 \mathrm{pc}$. For simplicity the sound speed in clouds is assumed to follow an $r^{-1 / 4}$-law. The radial distribution follows from Eq. (30) and the vertical distribution is described in Sect. 3.2. The mass accretion rate in the torus is taken to $\dot{M}=20 \dot{M}_{\mathrm{Edd}}$ for a $1.2 \times 10^{7} M_{\odot}$ black hole and corresponds to the solid-lines in Figs. 2 and 3.

The mass density distribution from Eq. (18) can be used to construct a gravitational potential by solving the Poisson equation. The Potential $\Phi(r)$ to be used for the relative potential $\Psi(r)$ (Eq. (14)) which includes a central black hole of mass $M_{\mathrm{BH}}$, reads

$$
\begin{aligned}
\Phi(r)= & -\frac{G M_{\mathrm{BH}}}{r}+\frac{2}{3} \pi G \rho_{*, \mathrm{c}} r^{2} \\
& \times\left[3{ }_{2} F_{1}\left(\frac{1}{2}, \frac{3}{2 \alpha}, 1+\frac{1}{\alpha},-\left(\frac{r}{R_{\mathrm{c}}}\right)^{2 \alpha}\right)\right. \\
& \left.-2{ }_{2} F_{1}\left(\frac{1}{2}, \frac{3}{2 \alpha}, 1+\frac{3}{2 \alpha},-\left(\frac{r}{R_{\mathrm{c}}}\right)^{2 \alpha}\right)\right] .
\end{aligned}
$$

For radii much larger than the core radius $R_{\mathrm{c}}$ the series expansion of the hypergeometric functions ${ }_{2} F_{1}$ shows that the stellar contribution to the potential varies as $\Psi(r) \propto\left(r / R_{\mathrm{c}}\right)^{2-\alpha}$. This is consistent because the gravitational force vanishes in an isothermal cluster $(\alpha=2)$.

\subsection{The surface density}

The vertical structure discussed in Sect. 3.2 is a two-parameter family of velocity dispersion $\sigma$ and cut-off height $x_{H} R$. For $\sigma \ll \sigma_{\mathrm{c}}$ with

$\sigma_{\mathrm{c}}=\frac{0.8 x_{H}}{\sqrt{2 \pi}} R \Omega$ 

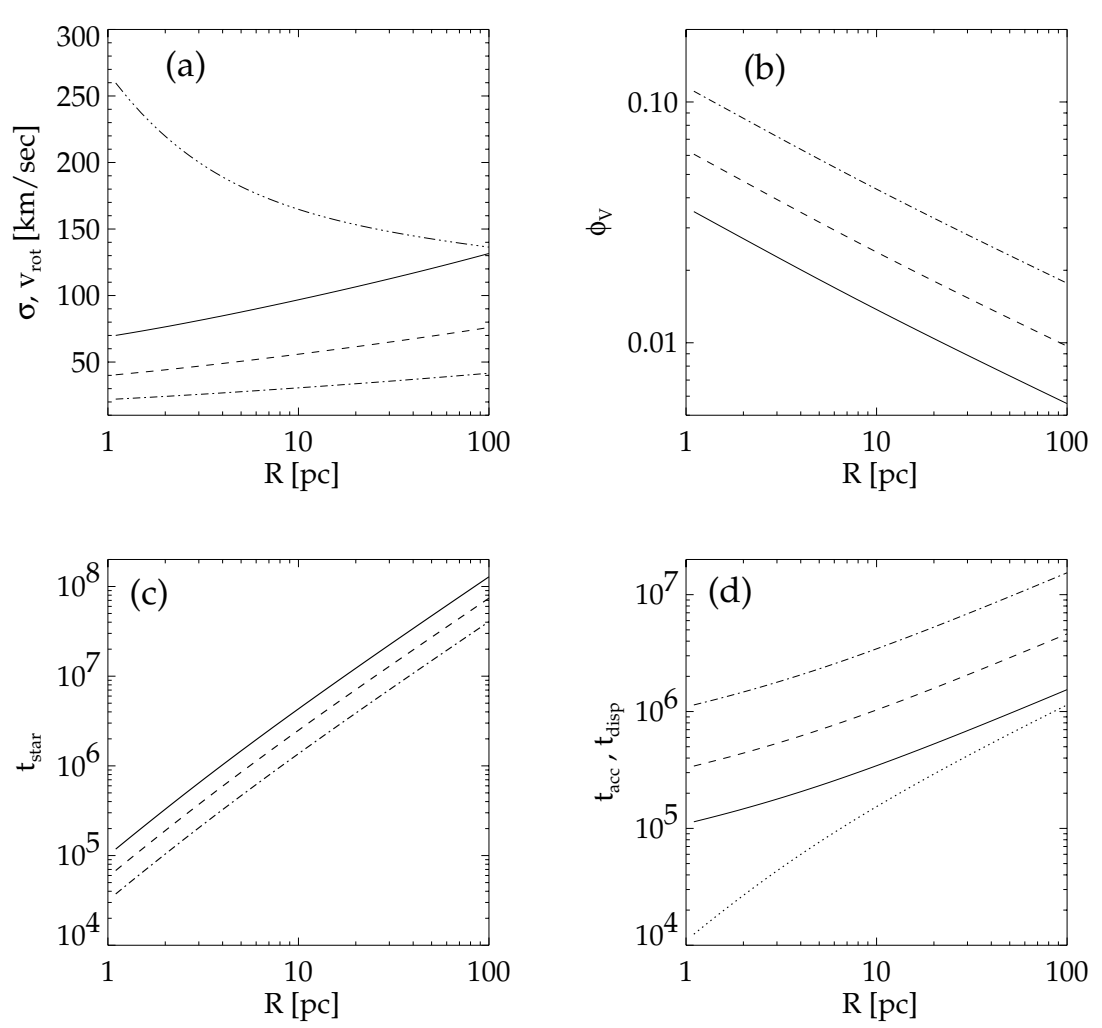

Fig. 2. The radial structure of the torus described in Sect. 4.1 with the mass distribution from Sect. 6 for NGC 1068 with $\tau=1$ and $Q$ at its local maximum (Eq. (27)). Three different accretion rates are assumed $\dot{m}=$ [30 (solid line), 10 (dashed), 3 (dashdotted)], where $\dot{m}$ is the accretion rate in units of the Eddington rate for the central black hole. On the top left a) the velocity dispersion $\sigma$ can be compared to the Keplerian circular velocity (dashed- $3 \times$ dotted). The top right $\mathbf{b}$ ) shows the volume filling fraction of dusty clouds $\phi_{V}$ to be in the range of $0.5 \%$ to $10 \%$. The star formation time-scale (Eq. (44)) is plotted bottom left c) which smaller than the accretion timescale $t_{\text {acc }}$ (Eq. (42)) displayed bottom right d) only in the innermost region. Plot d) also gives the dissipation or collapse time (Eq. (45)) in the absence of heating (dotted) for $\dot{M}=30 \dot{m}$, which is the shortest timescale considered. the vertical density distribution is almost isothermal with a typical scale height $H=\sigma / \Omega<x_{H} R$. In that regime the surface density is well approximated by

$\Sigma=\xi \rho_{0} H=\sqrt{2 \pi} \rho_{0} H$

where $\rho_{0}=\rho(z=0)$ is the midplane density. In the regime of small $\sigma$ we find the expected relation between central density, scale height and surface density.

For $\sigma>\sigma_{\mathrm{c}}$ the surface density saturates at about

$\Sigma_{\text {sat. }}=0.8 \rho_{0} x_{H} R$

and is therefore independent of $\sigma$. The density distribution is not isothermal anymore and its variation vertically is weak until it cuts off steeply when approaching $x_{H} R$. The height of the torus bears no relation to the scale height $\sigma / \Omega$. In the model discussed in the following we will use $\sigma=f_{\sigma} \sigma_{\mathrm{c}}$ with $f_{\sigma}=0.7$ so that Eq. (23) is valid and a fair approximation for the true surface density, while the approximations Eqs. (23) and (24) overestimate $\Sigma$ particularly for $\sigma \approx \sigma_{\mathrm{c}}$.

\section{Angular momentum transport and accretion}

Like in ordinary accretion disks, the effective viscosity from Eq. (2) allows mass to be accreted towards the black hole. The angular momentum barrier is overcome by most of the clouds through redistribution of angular momentum described by

$v \Sigma \frac{\partial \Omega}{\partial R}=-\frac{\dot{M}}{2 \pi R} \Omega$

in a stationary situation. Here $\dot{M}$ is the total mass accretion rate, which we assume to be constant at all radii throughout the torus. From Eqs. (25) and (2) we get the velocity dispersion in terms of the accretion rate in the form

$\sigma=R \Omega\left(\frac{Q\left(1+\tau^{2}\right)}{2 \tau \beta t_{\mathrm{g}}} \frac{\Omega}{\kappa}\right)^{1 / 3}$

with the definition of the logarithmic derivative $\beta=$ $-\partial(\ln \Omega) / \partial(\ln R)$ and the dimensionless growth time $t_{\mathrm{g}}=$ $\Omega M(R) / \dot{M}$ for the enclosed total mass at radius $R$. The choice of $t_{\mathrm{g}}$ is particularly useful for an isothermal stellar cluster ( $\alpha=2$ ), where $t_{\mathrm{g}}$ is constant.

Inserting the velocity dispersion (Eq. (26)) from the accretion process into the shear limit Eq. (7) gives

$Q \leq\left[\frac{8 \tau^{2}\left(1+\tau^{2}\right)}{\pi^{3} \xi^{3} \beta t_{\mathrm{g}}}\left(\frac{v_{\phi}}{c_{\mathrm{s}}}\right)^{3} \sqrt{\frac{2 \kappa}{\Omega}}\right]^{1 / 2}=Q_{\text {limit }}$,

where $v_{\phi}=R \Omega$ is the local rotation velocity of the torus. It is worth noticing that the shear limit on $Q$ has only a weak radial dependence because $v_{\phi}^{3} / t_{\mathrm{g}}=G \dot{M}$ is constant in a stationary state and the variation is due to the variation of the sound speed in clouds $Q_{\text {limit }} \propto c_{\mathrm{s}}^{-3 / 2}$. A weak additional dependency on the potential is introduced through the ratio $\sqrt[4]{2 \kappa / \Omega}$.

\subsection{Radial structure}

The density distribution in Fig. 1 and the radial variations of some characteristic quantities plotted in Figs. 2 and 3 make use of the particular choice of the cut-off scale $x_{H}$ from Sect. 3.4 and the assumption of clouds living at the shear limit. We now 

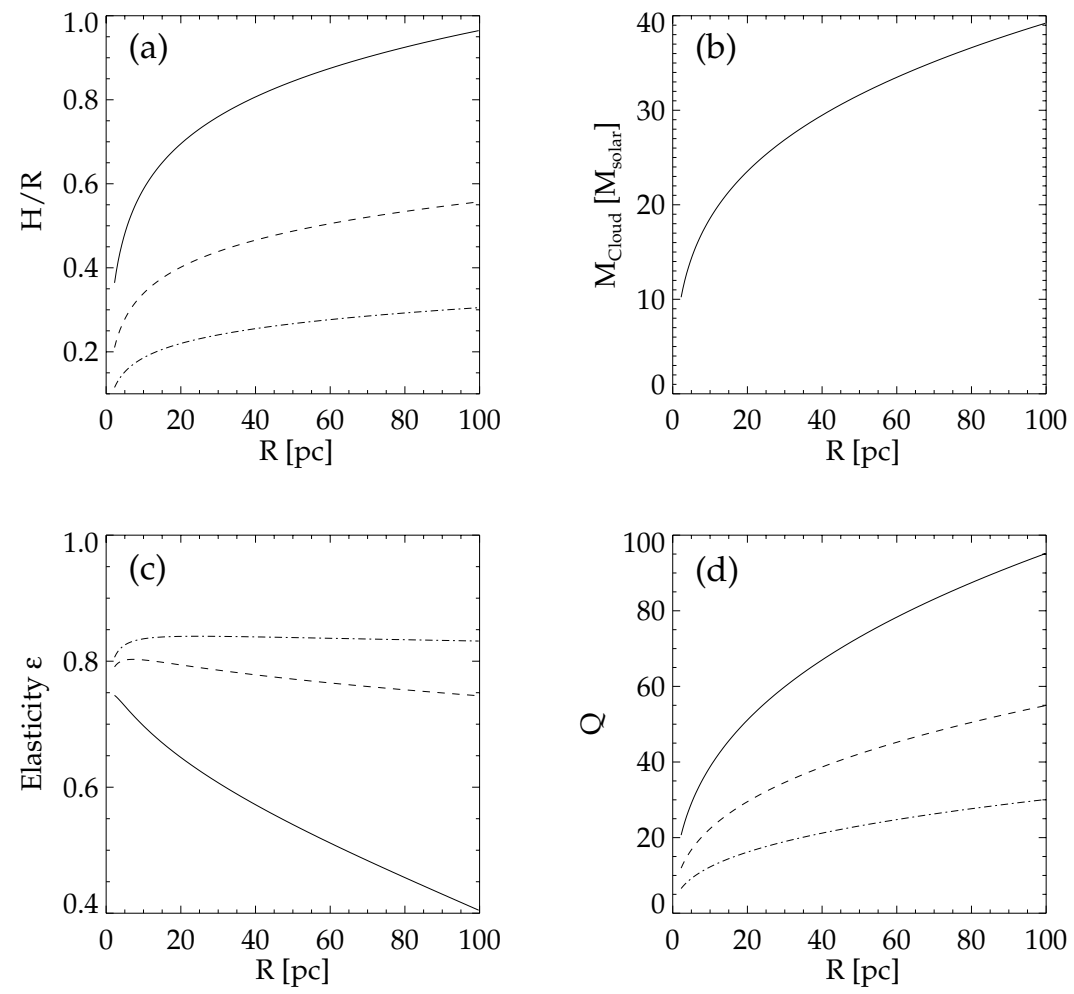

Fig. 3. For the same specific model assumed in Figs. 1 and 2 we plot the relative scale $H / R$ at the top left a) for the three accretion rates $\dot{m}=[30$ (solid line), 10 (dashed), 3 (dash-dotted)] as a function of radius. The cloud mass at top right $\mathbf{b}$ ) is seen to be independent of $\dot{m}$. The coefficient of restitution $\epsilon$ or elasticity in cloud collisions required by our model (Eq. (37)) is shown bottom left $\mathbf{c})$. The variation of the limiting value of Toomre- $Q$ is given bottom right $\mathbf{d}$ ).

summarize the functional dependencies of this model and start with the velocity dispersion

$\sigma=v_{\phi}\left(\frac{v_{\phi}}{c_{\mathrm{s}} t_{\mathrm{g}}} \frac{1+\tau^{2}}{\pi \xi \beta} \sqrt{\frac{2 \Omega}{\kappa}}\right)^{1 / 2}$.

For a thick torus the velocity dispersion must be a sizable fraction of the Keplerian rotation velocity, which implies that the factor $v_{\phi} /\left(c_{\mathrm{s}} t_{\mathrm{g}}\right)=\dot{M} R /\left(c_{\mathrm{s}} M(R)\right)$ must be of order unity. It follows directly that the mean free path for clouds

$l_{\text {coll }}=\tau R \frac{\sigma}{v_{\phi}}=R\left(\frac{v_{\phi}}{c_{\mathrm{s}} t_{\mathrm{g}}} \frac{\tau^{2}\left(1+\tau^{2}\right)}{\pi \xi \beta} \sqrt{\frac{2 \Omega}{\kappa}}\right)^{1 / 2}$

is comparable to $\tau R$ for a thick torus. For the central density we find

$\rho_{0}=\frac{M(R)}{2 R^{3}}\left[\frac{\xi \pi t_{\mathrm{g}} \beta}{\tau^{2}\left(1+\tau^{2}\right)}\left(\frac{c_{\mathrm{s}}}{v_{\phi}}\right)^{3}\right]^{1 / 2}\left(\frac{\kappa}{2 \Omega}\right)^{3 / 4}$,

which shows that the cloud mass density in the torus is approximately $\left(c_{\mathrm{s}} / v_{\phi}\right)^{3 / 2}$ times the stellar mass density divided by $\tau^{2}$. The volume filling factor of clouds turns out to be

$\phi_{V}=\left(\frac{\pi^{3} \xi \beta}{9 \tau^{2}\left(1+\tau^{2}\right)} t_{\mathrm{g}}\left(\frac{c_{\mathrm{s}}}{v_{\phi}}\right)^{3} \sqrt{\frac{2 \Omega}{\kappa}}\right)^{1 / 2}$

and the mass of individual clouds is

$M_{\text {Cloud }}=M(R) \frac{\pi^{3}}{16}\left(\frac{c_{\mathrm{s}}}{v_{\phi}}\right)^{3} \sqrt{\frac{\Omega}{2 \kappa}}$.

The cloud mass does not directly depend on the mass accretion rate, but is a strong function of the sound speed within the clouds. The surface density of this particular torus model is

$\Sigma=\frac{M(R)}{2 R^{2}} \frac{\xi}{\tau} \frac{c_{\mathrm{S}}}{v_{\phi}} \sqrt{\frac{\kappa}{2 \Omega}}$, which is also independent of $\dot{M}$ and proportional to the stellar surface density times $c_{\mathrm{s}} /\left(\tau v_{\phi}\right)$.

The torus is energetically powered by accretion and the corresponding radial accretion velocity is

$v_{\mathrm{acc}}=\frac{\dot{M}}{2 \pi r \Sigma}=\frac{\tau}{\xi \pi t_{\mathrm{g}}} \frac{v_{\phi}^{2}}{c_{\mathrm{s}}} \sqrt{\frac{2 \Omega}{\kappa}}$.

The comparison with the velocity dispersion from Eq. (28) shows that both velocities are of the same order for moderate to sparsely populated tori $\tau \geq 1$. Only very dense cloud distributions $\tau \ll 1$ with very short mean free paths for clouds will show the conventional behavior of accretion disks, where $v_{\text {acc }} \ll \sigma$. This approaches the hydrodynamic situation and the velocity dispersion is then identified with the sound speed in the medium.

The large velocity dispersions and accretion velocities, which are a direct consequence of the geometric thickness of the torus, hint at possible sub-Keplerian rotation of the flow. For the discussion so far, Keplerian rotation was assumed, which is valid if no other comparable terms besides the gravitational and the centrifugal force in the radial momentum balance appears. While the advective term $v_{\text {acc }}\left(\partial v_{\text {acc }} / \partial R\right)$ is weak in the region of a isothermal stellar cluster, the pressure gradient $\rho_{0}^{-1} \partial\left(\rho_{0} \sigma^{2}\right) / \partial R$ is not. This pressure term is approximately $\sigma^{2} / R=(H / R)^{2} R \Omega^{2}$ and supports the torus against gravity. SubKeplerian rotation of the order

$\Omega=\Omega_{\mathrm{K}} / \sqrt{1+(H / R)^{2}}$

is to be expected, but the corrections are small as long as $H / R<$ 0.7 , which is only violated for the torus model with $\dot{m}=30$ at large radii. 
So far we have not made use of an energy equation, where we have to balance the kinetic energy losses due to cloud collisions and an advective energy transport with gains from mass accretion in the potential of black hole and stars. The collisions will be partly elastic and we use the coefficient of restitution $\epsilon$, which parameterizes the loss of tangential momentum in the collisions (e.g. Goldreich \& Tremaine 1978), to derive an energy balance

$$
\begin{aligned}
v_{\mathrm{acc}} \Sigma \frac{\partial\left(3 \sigma^{2} / 2\right)}{\partial R} & -v_{\mathrm{acc}} \sigma^{2} R \frac{\partial(\Sigma / R)}{\partial R}= \\
& -v \Sigma \beta^{2} \Omega^{2}+\frac{4}{\sqrt{\pi}}\left(1-\epsilon^{2}\right) \frac{\Sigma \sigma^{3}}{l_{\text {coll }}} .
\end{aligned}
$$

The energy loss in collisions is calculated for an isotropic, Maxwellian velocity distribution following Goldreich \& Tremaine (1978). The collision rate is quadratic in the number density of clouds and we have corrected for this the vertically integrated form of Eq. (36) by an additional factor $1 / 2$ in the last term. Neglecting variations of $\kappa / \Omega$ and $\beta$ we can solve this balance and find with the assumption of an $c_{\mathrm{S}} \propto R^{-1 / 4}$-law

$\epsilon^{2}=1-\frac{\sqrt{\pi}}{4}\left(\frac{\beta^{2} \tau^{2}}{1+\tau^{2}}+\frac{\tau v_{\mathrm{acc}}}{v_{\phi}}\left(\frac{11}{8}-\frac{\partial \ln \frac{M(R)}{R}}{2 \partial \ln R}\right)\right)$.

Depending on the uncertain physics of cloud-cloud collisions we can only constrain the coefficient of restitution to the interval $0<\epsilon<1$. The resulting $\epsilon$ for our specific model for the mass distribution in NGC 1068 (see Sect. 6) is shown in Fig. 3c.

The range of $0.4<\epsilon<0.85$ found in our models is in reasonable agreement with the situation investigated by Goldreich $\&$ Tremaine (1978) where they used an anisotropic velocity distribution and neglected advective effects, which are important at large mass accretion rates and provide an effective local cooling process. The surprising result is that tori with high accretion rates are geometrically thick in spite of the smaller elasticity of collisions. This a due to the positive entropy gradient in the distribution of clouds (left side of Eq. (36)) so that an increased inward mass transport allows collisions to transfer more specific entropy from the random motion of clouds to internal heat.

The large coefficient of restitution implied for cloud collisions might be provided by magnetic fields in the clouds. An in depth analysis of cloud collisions in the environment of an AGN is required to test our model predictions on $\epsilon$ or to infer a value of $\tau$, the collision time, for a given radius and mass accretion rate.

\subsection{Scaling laws}

The near- to mid-infrared radiation from the torus will be dominated by emission from the innermost region, where the influence of the black hole is strongest. Optically thin mid- to farinfrared emission will come from areas where the mass of the stellar cluster is dominant and where most of the mass of the torus is located. Outside the core radius of the cluster, where the stellar mass density decreases as $r^{-\alpha}(1<\alpha<3)$, we can give simple scalings with radius and sound speed. All the uncertain physics is hidden in the sound speed, which depends on the heating by collisions, direct and indirect heating by hard AGN radiation, radiative cooling, and pressure balance with the inter-cloud medium. From the Eqs. (28)-(34) we summarize the scaling laws:

$$
\begin{array}{ll}
t_{\mathrm{g}} \propto R^{3-3 \alpha / 2} & \sigma \propto c_{\mathrm{s}}^{-1 / 2} \\
l_{\text {coll }} \propto c_{\mathrm{s}}^{-1 / 2} R^{\alpha / 2} & \Sigma \propto c_{\mathrm{s}} R^{-\alpha / 2} \\
M_{\text {Cloud }} \propto c_{\mathrm{s}}^{3} R^{\alpha / 2} & \phi_{V} \propto c_{\mathrm{s}}^{3 / 2} \\
v_{\text {acc }} \propto c_{\mathrm{s}}^{-1} R^{\alpha / 2-1} & \rho_{0} \propto c_{\mathrm{s}}^{3 / 2} R^{-\alpha} .
\end{array}
$$

It is important to note that the mean free path for clouds grows not as fast as would be guessed from the central density alone. The steep mass density gradient is partly compensated by an increased cloud radius $r_{\mathrm{Cl}} \propto c_{\mathrm{s}} R^{\alpha / 2}$. The required coefficient of restitution in cloud collisions in our model (Eq. (37)) depends on the ratio $v_{\text {acc }} / v_{\phi} \propto \dot{M} c_{\mathrm{s}}^{-1}$ and $\beta^{2}$. In our scenario the changes at small radii arise from the transition in the potential at the sphere of influence of the black hole, while at large radii changes are due to the assumed dependence on $R$ of the sound speed in clouds $c_{\mathrm{s}} \propto R^{-1 / 4}$ as can be seen in Fig. 3c.

The size of clouds (Eq. (6)) and their mass (Eq. (32) and Fig. 3b) is independent of the mass accretion rate and show a trend of decreasing size and mass when approaching the black hole. This is consistent with clouds being disrupted in collisions, so that an increasing number of smaller and smaller clouds are transported through the torus.

\subsection{Time-scales}

To estimate the life time and possible variability of the torus we collect the main time-scales connected to the overall structure. Fluctuations due to the small number of clouds responsible for processes like the opacity along a particular line of sight, e.g. to the central black hole, can occur on much shorter time intervals.

In our scenario viscosity generates accretion towards the center on the viscous time-scale

$t_{\mathrm{acc}}=\frac{R^{2}}{v}=\frac{\pi \xi \beta}{\tau} \frac{M(R)}{\dot{M}} \frac{c_{\mathrm{s}}}{v_{\phi}} \sqrt{\frac{\kappa}{2 \Omega}} \propto \dot{M}^{-1} c_{\mathrm{s}} R^{2-\alpha / 2}$,

where the scaling with radius on right in the proportionality is for a stellar cluster as in Sect. 4.2. The mass consumed in star formation is locked in stars until part of it is regained from stellar winds and supernovae. We parameterize the star formation rate following Vollmer \& Beckert (2003)

$\dot{\Sigma}_{\star}=\phi_{V} \rho_{0} \sigma$

and derive a consumption time-scale for torus mass by

$t_{\star}=\frac{\Sigma}{\dot{\Sigma}_{\star}}=\frac{\tau}{\phi_{V} \Omega} \propto \sqrt{\dot{M}} c_{\mathrm{s}}^{-3 / 2} R^{\alpha / 2}$

with the same assumption for the scaling law on the right as in Eq. (42). Another relevant time-scale is the dissipative lifetime of the torus in the absence of reheating. We estimate it as the ratio of randomized kinetic energy of clumps $3 \sigma^{2} / 2$ to the energy loss rate in collisions $\frac{4}{\sqrt{\pi}}\left(1-\epsilon^{2}\right) \sigma^{2} / l_{\text {coll }}$ and get

$t_{\text {disp }}=\frac{3 \sqrt{\pi} \tau}{4\left(1-\epsilon^{2}\right) \Omega}$ 
which is much longer than the orbital time only for sparsely populated tori with $\tau \gg 1$.

In Figs. $2 \mathrm{c}$ and $2 \mathrm{~b}$ we compare the accretion with the star formation time-scale and the collapse time for the case of NGC 1068 for different mass accretion rates. The typical star formation time-scale $t_{\star}$ at a radius of $10 \mathrm{pc}$ is a few times $10^{6}$ years. Apart from the innermost region the accretion time is smaller than the star formation time in models with high mass accretion rates considered here. If our parameterization of star formation is correct the accretion process is faster than the time needed for effective star formation and this justifies our assumption that star formation and subsequent supernovae are unimportant for the dynamics of the torus. The short collapse or dissipative lifetime in these inner regions probably requires a more accurate energy balance than presented here.

\section{Obscuration by the torus}

The well constrained model of a torus developed in the preceding sections determines the orientation dependent obscuration required for a successful unification of Seyfert and quasar nuclei of types 1 and 2 . We consider two possible definitions of an obscuration angle, measured from the torus midplane, for our cloud distribution.

Our first definition of an obscuration angle $i_{N}$ is the inclination for which we have on average $N\left(i_{N}\right)=1$ or any other number of clouds along a line of sight to the central AGN. For a given inner and outer radius of the torus the number of clouds as a function of angle $i$ measured from the midplane is

$N(i)=\int_{s_{\text {sub }}}^{s_{\text {out }}} \frac{\mathrm{d} s}{l_{\text {coll }}}=\cos ^{-1} i \int_{s_{\text {sub }} \cos i}^{s_{\text {out }} \cos i} \frac{\mathrm{d} R}{l_{\text {coll }}}$

The mean free path is found from Eq. (29) in the midplane and the reduced density from the vertical structure discussed in Sect. 3.2. For the actual calculation we use $\tau=1$ and the model of the gravitational potential of NGC 1068 (see Sect. 6) as a representative example. The sound speed is assumed to follow a $c_{\mathrm{s}} \propto R^{-1 / 4}$-law with $c_{\mathrm{s}}$ approximately $3 \mathrm{~km} \mathrm{~s}^{-1}$ at the sublimation radius (inner boundary) of $R=1 \mathrm{pc}$. The free parameter is thus $t_{\mathrm{g}}$ or equivalently the mass accretion rate in the torus, which we specify in terms of the Eddington rate for the assumed $1.2 \times 10^{7} M_{\odot}$ black hole of NGC 1068. Equation (46) can be inverted numerically to find the obscuration angle $i_{N}$ and the results are plotted in Fig. 4 for finding $N$ between 1 and 6 clouds along the line of sight to the center.

The second criterion for an obscuration angle is by the mean hydrogen column density in clouds along a line of sight

$N_{\mathrm{H}}(i)=\int_{s_{\text {sub }}}^{s_{\text {out }}} \frac{\mathrm{d} s}{l_{\text {coll }}} N_{\mathrm{H}, \mathrm{Cl}}$,

which is the equivalent to Eq. (46) including the column density. The column for an individual cloud is

$N_{\mathrm{H}, \mathrm{Cl}}=\frac{\kappa \sigma}{\pi \xi \tau Q \mu m_{\mathrm{H}} G}$.

Here the mean column scales as $N_{\mathrm{H}, \mathrm{Cl}} \propto c_{\mathrm{S}} R^{-\alpha / 2}$ and is independent of the mass accretion rate. The resulting $i_{\text {Obsc }}$ of

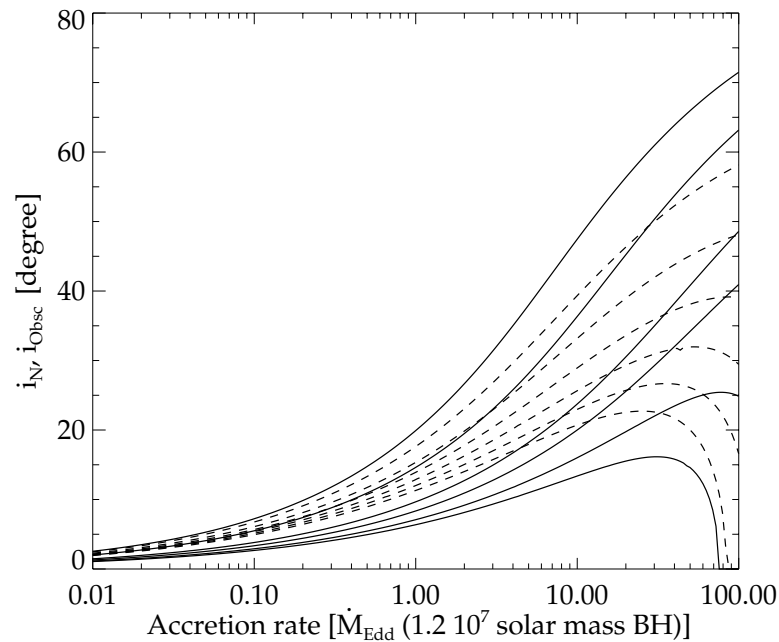

Fig. 4. The obscuration angle $i_{N}$ and $i_{\text {Obsc }}$ as function of mass accretion rate derived from the model adapted for NGC 1068 with vertical density structure given by Eq. (15). The outer radius of the torus is set to $R_{\text {out }}=100 \mathrm{pc}$. The dashed lines show the angle $i_{N}$ for having on average $N=[1,2,3,4,5,6]$ clouds along the line of sight. Due to the vertical stratification of the torus the probability of seeing more clouds increases for smaller angles and the dashed lines are ordered accordingly. The solid lines show the obscuration angle $i_{\text {Obsc }}$ for finding an average hydrogen column density of $N_{\mathrm{H}}=$ $\left[10^{22}, 10^{23}, 10^{24}, 2 \times 10^{24}, 4 \times 10^{24}, 6 \times 10^{24}\right]$ per $\mathrm{cm}^{2}$. Again the stratification leads to an increased column for smaller angles and the identification of lines with column density is obvious.

the inversion for various column densities of $N_{\mathrm{H}}\left(i_{\mathrm{Obsc}}\right)$ is also shown in Fig. 4. If an obscuration angle $i_{N=1}$ above $40^{\circ}$ is required for the average Seyfert nucleus, this implies an accretion rate 10 times larger than the Eddington rate for the corresponding black hole. These average Seyfert nuclei seen as type 2's at an inclination $i<20^{\circ}$ will have hydrogen columns $N_{\mathrm{H}}>10^{24} \mathrm{~cm}^{-2}$ in the clouds alone. But the number of clouds along the line of sight to the center is only between 6 and 10 , so that substantial fluctuations are expected.

A key parameter for the success of the clumpy radiative transfer of Nenkova et al. (2002) is the mean number of clouds along a line of sight to the center in the torus midplane. For the specific case of the NGC 1068 torus we find from Eq. (46) $N \approx 12$ at $\dot{M}=20 \dot{M}_{\text {Edd }}$ and from Eq. (29) one sees that $N(i=$ 0 ) is a moderate function the mass accretion rate: $N \propto \dot{M}^{-1 / 2}$. The range preferred by Nenkova et al. (2002) of 5 to 10 clouds corresponds to $\dot{M} / \dot{M}_{\mathrm{Edd}}=30$ to 120 . Due to the reduced scale height in the inner, black hole influenced, region of the torus the integral in Eq. (46) is dominated by contributions from small radii.

An estimate for the mass lost in an outflow from the inner region of a torus is given by Krolik \& Kriss (2001) and shows that the ratio $\dot{M}_{\text {out }} / \dot{M}_{\text {acc }}$ can reach $\sim 100$. This estimate refers to an ionization parameter $\xi=100$, a wind velocity of $500 \mathrm{~km} \mathrm{~s}^{-1}$, and a covering fraction of wind material around the nucleus of $\sim 1 / 6$. An ionizing radiation efficiency of $5 \%$ for the accretion flow into the black hole is assumed to relate accretion rate to the ionizing luminosity. It follows that substantially more mass 
can be transported through the torus and lost in an outflow than is actually swallowed by the black hole.

\section{The torus in NGC 1068}

We apply our model to the proposed torus around the AGN of the extensively studied Seyfert 2 Galaxy NGC 1068. The $M_{\mathrm{BH}}-\sigma_{\text {bulge }}$-relation predicts a black hole mass of about $8 \times$ $10^{6} M_{\odot}$, where we used the slope and normalization by Tremaine et al. (2002). Some of the observed $\mathrm{H}_{2} \mathrm{O}$-maser spots (Greenhill \& Gwinn 1997) around the radio core component S1 (Gallimore et al. 2001), which corresponds to component 2 in earlier MERLIN maps (Muxlow et al. 1996) trace a rotating disk or ring. The maser velocities appear inconsistent with Keplerian rotation around a central point mass and the rotation velocity falls off with radius as $\propto r^{-0.35}$ (Kumar 1999). Neglecting non-gravitational forces, the enclosed mass at $0.65 \mathrm{pc}$ from the center is $1.5 \times 10^{7} M_{\odot}$. The rotation profile can be due to a massive disk (Huré 2002), but we follow Kumar (1999) and find a reasonable model with a black hole mass of $1.2 \times 10^{7} M_{\odot}$ and a strongly concentrated stellar cluster with $\alpha=2.1$, a core radius of $0.32 \mathrm{pc}$ and a core density of $\rho_{\star, \mathrm{c}}=5.25 \times 10^{6} M_{\odot} \mathrm{pc}^{-3}$. Core radius and density are comparable to the stellar cluster centered on Sgr A* (Schödel et al. 2003), the radio source at the dynamical center of our Galaxy. The stellar core mass used for NGC 1068 is only 1.4 times larger in spite of the four times larger black hole mass. The dusty torus beyond $2 \mathrm{pc}$ is dynamically dominated by the potential of the stellar cluster in our assumed mass distribution.

The currently estimated AGN-luminosity of NGC 1068 is $L_{\text {bol }}=8 \times 10^{44} \mathrm{erg} / \mathrm{s}$ (Pier et al. 1994), which corresponds to mass accretion at about one third of the Eddington rate $(10 \%$ radiation efficiency) for our mass estimate. In addition to the radiated luminosity, the AGN also drives a jet and an outflow. From the estimate of the mass outflow rate at the end of Sect. 5 an accretion rate through the torus of $\sim 30$ times the Eddington accretion rate of the black hole seems possible. This is also the rate required to raise the torus obscuration angle to $i_{N=1} \sim 50^{\circ}$ and is thus consistent with the aspect angle dependent unification of type 1 and type 2 AGN. While Crenshaw \& Kraemer (2000) derive velocities in the NLR of NGC 1068 of more than $1000 \mathrm{~km} / \mathrm{s}$ far from the nucleus, Ogle et al. (2003) find smaller velocities $\sim 400 \mathrm{~km} \mathrm{~s}^{-1}$ and covering factors of $6 \%$ in the electron scattering region, but small ionization parameters $\xi \leq 10$ in X-ray NLR clouds. Both observations support high mass outflow rates and therefore high accretion rates in the torus.

\subsection{The extended stellar cluster in the nucleus of NGC 1068}

From $H$ and $K$-band observations of $\mathrm{CO}$ and Si features Thatte et al. (1997) derive a surface brightness profile of stellar light from late type stars corrected for dilution by hot dust from within $2^{\prime \prime}$ of the nucleus. The surface brightness profile is consistent with a power-law $S \propto r^{1-\alpha^{\prime}}$ with $\alpha^{\prime}=1.92 \pm 0.07$ between $0.3^{\prime \prime}$ and $4^{\prime \prime}$ in radius. The central cluster extends therefore out to $250 \mathrm{pc}$ and the dusty torus lives in the gravitational potential of this cluster.
Form velocity dispersion measurements Dressler (1984) of $\sigma_{\star} \sim 145 \mathrm{~km} \mathrm{~s}^{-1}$ one derives an upper limit of $6.5 \times 10^{8} M_{\odot}$ within $1^{\prime \prime}$ while the extension of the central cluster in our model with $\alpha=2.1$ contributes $3.2 \times 10^{8} M_{\odot}$.

If the stellar light distribution traces mass we expect an enclosed mass profile $M_{\star}(r) \propto r^{3-\alpha^{\prime}}$ and the circular velocity curve $v_{\phi} \propto r^{1-\alpha^{\prime} / 2}$ rises outwards. Under the the assumption that the slope of the central cluster equals the inferred slope at larger radii $\alpha=\alpha^{\prime}$ the circular velocity rises to $205 \mathrm{~km} \mathrm{~s}^{-1}$ at $1^{\prime \prime}$.

The rotation curve at even larger radii from Garcia-Lorenzo et al. (1997) is consistent with this upper limit for inclinations less than $i_{\max } \sim 45^{\circ}$. When the inner torus and the outer stellar disk are in the same plane, an inclination of $i \approx 20^{\circ}$ is more likely and the rotation velocity is about $150 \mathrm{~km} \mathrm{~s}^{-1}$ in the torus region with an enclosed mass of $\sim 3.8 \times 10^{8} M_{\odot}(\alpha=2.06)$ within $1^{\prime \prime}$. The stellar mass distribution used in our model for the central cluster leaves therefore room for additional mass traced at larger radii and is close to isothermal.

\section{Summary}

We developed a dynamical model for the radial and vertical structure of a clumpy torus around an AGN based on the ideas described in Vollmer et al. (2004). The vertical stratification of arbitrarily thick rotating cloud distributions in the potential of galactic nuclei is derived under the assumption of hydrostatic equilibrium. For modified isothermal distributions we present cloud number densities depending on the vertical scale height $H$ and a cut-off height $x_{H}$. Such a configuration with cut-off can be produced by a massive outflow from the central regions of AGNs.

The derived vertical structure is combined with an accretion scenario for a geometrically and optically thick torus surrounding the AGN. We show that large mass accretion rates in the torus will produce obscuring cloud distributions with scale heights $H>0.5 R$. The mean number of clouds along particular lines of sight to the center are calculated and it is found that for angles $>45^{\circ}$ w.r.t. the torus midplane a line of sight to the AGN may intercept, on average, one cloud.

The thickness of the cloud distribution is supported by randomizing the ordered rotation velocities in the torus. Cloud collisions will destroy clouds and produce new ones leading to more smaller clouds at small radii. We describe the appearance of the resulting distribution by considering only the largest clouds present. The size of clouds is limited by tidal shear, where larger clouds are not sufficiently kept together by selfgravity. These large clouds determine the mean free path between clouds and the optical depth for photons. An optically thick torus at optical and NIR wavelength requires massive clouds ( $>10 M_{\odot}$ in our model) and a mean free path not much larger than $H$, so that the collision time for clouds is about the dynamical time of the torus $\Omega^{-1}$. The thickness of the torus implies a coefficient of restitution $\epsilon$ between 0.4 and 0.85 depending on the mass accretion rate, which itself is a result of the cloud collisions.

We use the prototypical Seyfert 2 nucleus of NGC 1068 as a testbed for our model. The torus in the combined potential of 
a $1.2 \times 10^{7} M_{\odot}$ black hole and a nuclear stellar cluster will have the typical thickness of tori expected from the unified scheme of AGN for mass accretion rates above ten times the Eddington accretion rate of the black hole. Taking NGC 1068 as representative of partly obscured bright AGN, one can conclude that all obscured AGN must generate outflows to get rid of a large fraction of the mass transported through the torus.

The dynamical considerations for a cloudy torus are consistent with the model assumed by Nenkova et al. (2002) as the basis of their radiative transfer treatment for mass accretion rates of 30 times the Eddington rate for the black hole. The huge mass accretion rates through the torus are necessarily accompanied by massive outflows driven by photoevaporation at the inner rim of the torus. Only a small fraction of the mass eventually reaches the black hole but is sufficient to generate the ionizing luminosity. Detailed radiative transfer calculations using the derived cloud distribution presented here will produce simulated images and SEDs, which will allow observational tests of our model.

Acknowledgements. We wish to thank Bernd Vollmer, Werner Tscharnuter and Gerd Weigelt for stimulating discussions. Part of this work was support by the DFG through grant SFB439 (W.J.D.).

\section{References}

Abramowitz, M., \& Stegun, I. A. 1972, Handbook of Mathematical Functions (New York: Dover)

Alonso-Herrero, A., Quillen, A. C., Rieke, G. H., Ivanov, V. D., \& Efstathiou, A. 2003, AJ, 126, 81

Antonucci, R. R. J., \& Miller, J. S. 1985, ApJ, 297, 621
Binney, J., \& Tremaine, S. 1987, Galactic dynamics (Princeton, NJ: Princeton University Press), 747

Crenshaw, D. M., \& Kraemer, S. B. 2000, ApJ, 532, L101

Dressler, A. 1984, ApJ, 286, 97

Gallimore, J. F., Henkel, C., Baum, S. A., et al. 2001, ApJ, 556, 694

Garcia-Lorenzo, B., Mediavilla, E., Arribas, S., \& del Burgo, C. 1997, Ap\&SS, 248, 43

Goldreich, P., \& Tremaine, S. D. 1978, Icarus, 34, 227

Granato, G. L., Danese, L., \& Franceschini, A. 1997, ApJ, 486, 147

Greenhill, L. J., \& Gwinn, C. R. 1997, Ap\&SS, 248, 261

Huré, J.-M. 2002, A\&A, 395, L21

Krolik, J. H., \& Kriss, G. A. 2001, ApJ, 561, 684

Kumar, P. 1999, ApJ, 519, 599

Maiolino, R., \& Rieke, G. H. 1995, ApJ, 454, 95

Miller, J. S., \& Antonucci, R. R. J. 1983, ApJ, 271, L7

Muxlow, T. W. B., Pedlar, A., Holloway, A. J., Gallimore, J. F., \& Antonucci, R. R. J. 1996, MNRAS, 278, 854

Nenkova, M., Ivezić, Ž., \& Elitzur, M. 2002, ApJ, 570, L9

Ogle, P. M., Brookings, T., Canizares, C. R., Lee, J. C., \& Marshall, H. L. 2003, A\&A, 402, 849

Pier, E. A., \& Krolik, J. H. 1992, ApJ, 401, 99

Pier, E. A., \& Krolik, J. H. 1993, ApJ, 418, 673

Pier, E. A., Antonucci, R., Hurt, T., Kriss, G., \& Krolik, J. 1994, ApJ, 428, 124

Rowan-Robinson, M. 1995, MNRAS, 272, 737

Schödel, R., Ott, T., Genzel, R., et al. 2003, ApJ, 596, 1015

Schmitt, H. R., Antonucci, R. R. J., Ulvestad, J. S., et al. 2001, ApJ, 555,663

Thatte, N., Quirrenbach, A., Genzel, R., Maiolino, R., \& Tecza, M. 1997, ApJ, 490, 238

Tran, H. D. 2001, ApJ, 554, L19

Tremaine, S., Gebhardt, K., Bender, R., et al. 2002, ApJ, 574, 740

Vollmer, B., \& Beckert, T. 2003, A\&A, 404, 21

Vollmer, B., Beckert, T., \& Duschl, W. J. 2004, A\&A, 413, 949 